\title{
Nucleus Basalis Magnocellularis and Medial Septal Area Lesions Differentially Impair Temporal Memory
}

\author{
Warren H. Meck, ${ }^{1}$ Russell M. Church, ${ }^{2}$ Gary L. Wenk, ${ }^{3}$ and David S. Olton ${ }^{3}$ \\ 'Department of Psychology, Columbia University, New York, New York 10027, 2Department of Psychology, Brown \\ University, Providence, Rhode Island 02912, and '3epartment of Psychology, The Johns Hopkins University, Baltimore, \\ Maryland 21218
}

\begin{abstract}
Functional dissociations between the medial septal area (MSA) and the nucleus basalis magnocellularis (NBM) were examined using the concepts and experimental procedures developed by scalar timing theory. Rats were tested in variations of a signalled discrete-trial peak-interval schedule of reinforcement in which the response rate functions identified the time when the rats expected reinforcement. The variations assessed aspects of both reference and working memory for information obtained from prior trials and from the current trial. A double dissociation was found in reference memory. Rats with NBM lesions, like those with frontal cortex (FC) lesions, remembered the time of reinforcement as having occurred later than it actually did; rats with MSA lesions, like those with fimbria-fornix (FF) lesions, remembered the time of reinforcement as having occurred earlier than it did. A single dissociation was found in working memory. MSA lesions and FF lesions impaired working memory, while NBM and FC lesions had no effect on it. These data begin to identify the brain mechanisms underlying temporal memory; they indicate that the frontal and hippocampal systems are both involved, but in complementary ways; and they provide information that helps specify more clearly the functions of the frontal and hippocampal systems.
\end{abstract}

The basal forebrain cholinergic system (BFCS) has received considerable attention because of its involvement in Alzheimer's disease. Although the BFCS has a topologically organized set of projections throughout its extent, it has at the extremes 2 relatively distinct subcomponents, the nucleus basalis magnocellularis (NBM) and the medial septal area (MSA), which have projections to the frontal cortex (FC) and hippocampus (HIP), respectively (Schwarcz et al., 1979; Sofronien et al., 1982; Saper, 1984).

Neuropsychological investigations of the FC and HIP have described the functions of these 2 areas in very different terms (see O'Keefe and Nadel, 1978; Fuster, 1980; Milner, 1982; Rawlins, 1985, for reviews). Yet in animals, selective lesions of the NBM or MSA have produced similar behavioral impairments in all of the tasks that have been examined (see Olton et al., $1987 \mathrm{c}$; Wenk et al., 1987b, for reviews). This failure to find

\footnotetext{
Received Aug. 8, 1986; revised Apr. 27, 1987; accepted May 6, 1987.

This investigation was supported by Research Grants from the NIH for Biomedical Research Support (W.H.M.), and from National Institute of Mental Health Grants MH37049 (R.M.C.) and MH24213 (D.S.O.). The authors thank Brenda Cribbs and Lisa McCall for excellent technical assistance.

Correspondence should be addressed to Warren H. Meck at the above address. Copyright (C) 1987 Society for Neuroscience $0270-6474 / 87 / 113505-11 \$ 02.00 / 0$
}

functional dissociations is puzzling. On the one hand, it might reflect a true, functional homogeneity of the BFCS in spite of the functional heterogencity of its target sitcs. Altcrnatively, it might be an unintended outcome of the selection of behavioral tasks; selective lesions of the subcomponents of the hippocampal system (fimbria-fornix, CA1 and CA3 pyramidal cell layers, the hippocampus as a whole, etc.) and the frontal system (FC and NBM) have also failed to produce clear behavioral dissociations in tasks similar to the ones used to examine the behavioral effects of NBM lesions (see Olton et al., 1979; Kolb, 1984, for reviews).

A priori, a case can be made for the functional homogeneity or functional dissociation of the NBM and the MSA. Experimental tests of these 2 alternatives can be made only with behavioral tasks that provide unambiguous behavioral dissociations following lesions of the FC and the hippocampal system. The present experiment used this approach with procedures developed to assess timing behavior.

Scalar timing theory is a mathematical model that describes formal properties of the cognitive processes that may operate when an individual achieves a variety of temporal discriminations (Gibbon et al., 1984). This analysis has successfully accounted for more than $97 \%$ of the variance in behavior of both rats and pigeons in several different timing tasks. It has also led to the development of a set of experimental procedures that can be used to separate the different psychological processes involved in timing. In the basic discrete-trial fixed-interval (FI) procedure, each trial begins with a signal onset. The animal is free to respond at any time during the signal, but only the first response after the fixed duration is reinforced. The peak-interval (PI) procedure, a variation of the FI procedure, provides independent measures of several processes involved in temporal discriminations. Some trials are identical to those of the FI procedure. For other peak trials, however, the signal continues longer than its usual fixed duration in the FI procedure, and no reinforcement is available. During these nonreinforced peak trials, the animal's response rate increases as a function of time until a point near the time that reinforcement is available in the FI procedure, when it decreases. The time of the maximal response rate is called the peak time. Peak time and peak rate are independent measures, because treatments may affect each mcasure without affecting the other (Roberts, 1981).

Additional memory components can be added to the PI procedure by inserting a gap or retention interval. This peak interval with a gap (PI-GAP) procedure includes the usual FI and peak trials, as already described. It also has gap trials, in which the signal is turned on for an interval, off for an interval (creating 
a gap in the signal), and back on. Normal rats follow a stop rule during the gap trials (Meck et al., 1984, 1986b; Olton et al., $1987 \mathrm{a}, \mathrm{b})$. The rats stop timing during the gap, remember the signal duration prior to the gap, start timing again when the signal begins after the gap, and add the signal duration prior to the gap to the ongoing signal after the gap. Consequently, the peak time for the gap trials is shifted to the right by an amount of time equal to the interval of the gap (when animals are not timing).

In rats, lesions of the fimbria-fornix (FF), but not of the amygdala (AMG), produce 2 major changes in timing performance, as assessed by both the PI and the PI-GAP procedure (Olton et al., 1987a). In the PI procedure, during peak trials, rats with $F F$ lesions have a leftward shift in the timing function that is proportional to the time of reinforcement, indicating that they remember a significant event (food delivery) as having occurred earlier than it actually did. In the PI-GAP procedure, during the gap trials, rats with FF lesions fail to remember the duration of the stimulus prior to the gap and follow a reset rule rather than a stop rule. Following the gap, when the stimulus is turned back on, these rats respond as if the signal prior to the gap had not been presented. Consequently, the peak time during the gap trials is shifted to the right by an amount of time equal to the gap plus the signal duration prior to the gap. Taken together, these data indicate that following FF lesions, rats remember the time of reinforcement (a value obtained from reference memory) as being proportionally shorter than it actually was, and fail to remember trial-specific information about the duration of a previously timed event after a gap in the signal being timed (a value stored in working memory).

Preliminary evidence from unpublished studies (Marica, 1978) suggested that lesions of the FC had a different effect on timing behavior than did FF lesions. Of particular importance was the observation that $\mathrm{FC}$ lesions produced a rightward shift of the temporal discrimination function, opposite to the effect produced by FF lesions. Consequently, in addition to further analyzing of the behavioral effects of FC and FF lesions, the present experiment examined the behavioral sequellae of selective NBM and MSA lesions in the PI and PI-GAP timing tasks. If lesions of the NBM and MSA produce differential effects on the functioning of temporal memory, these results will provide evidence for the behavioral dissociation of these areas and identify some of the neural mechanisms involved in temporal discriminations. A report based on this work was presented previously (Meck et al., 1985).

\section{Materials and Methods}

\section{Subjects}

Experiments were performed on 40 male albino rats (350-400 gm) obtained from Charles River Breeding Laboratories. Each rat was housed individually throughout testing, with free access to water, and was maintained on a 12:12 hr lighv/dark cycle with fluorescent lights on at 6:00 AM. Each rat was fed a daily ration of $15 \mathrm{gm}$ of Agway Pro Lab Formula (RMH 3200), mixed with about $15 \mathrm{ml}$ of water, shortly after the experimental session.

\section{Apparatus}

Ten boxes $(23 \times 20 \times 22 \mathrm{~cm})$ with retractable response levers and associated equipment (e.g., pellet dispensers, house lights, and noise generators) were used; they have been described elsewhere in detail (Meck et al., 1984). A Noyes Precision food pellet $(45 \mathrm{mg})$ was used as reinforcement following an appropriate lever press. $\Lambda$ Digital Equipment
PDP-1 2 computer controlled the experimental equipment and recorded the data.

\section{Procedures}

Pretraining. Each rat received at least 2 sessions of combined magazine and lever training. During these sessions, a pellet of food was delivered once every $30 \mathrm{sec}$ and, in addition, each lever press produced food. The session began with the insertion of the left lever and continued until the rat had pressed the lever 60 times or until $30 \mathrm{~min}$ had passed, whichever came first. The house light was off during these and all subsequent sessions.

Preoperative baseline PI training (sessions 1-30). The 40 rats were divided into 4 groups of 10 rats each. Each group was trained for $3 \mathrm{hr}$ at the same time of day on alternate days. Sessions began at $8 \mathrm{AM}$ and 12 noon. Thus, 2 groups of rats were consistently trained during the 8 AM session and 2 groups of rats were consistently trained during the 12 noon session. A random half of the trials were $40 \mathrm{sec}$ FI trials. During these trials, food was made available $40 \mathrm{sec}$ after signal onset and the rat's first lever press after the fixed duration terminated the noise signal and activated the pellet dispenser. The remaining half of the trials were peak trials. During these trials, food was not available at $40 \mathrm{sec}$ and the noise signal continued for a total of $130 \mathrm{sec}$. Trials were separated by a $130 \mathrm{sec}$ intertrial interval (ITI). Data were collected for each rat only during peak trials.

Surgery. Ten rats, 5 trained at $8 \mathrm{AM}$ and 5 trained at 12 noon, were randomly selected for each of the 4 groups: MSA lesions, NBM lesions, FC lesions, and control operations.

The procedure for producing the lesions has been described in detail elsewhere (Mitchell et al., 1982; Hepler et al., 1985a, b). Each rat was given atropine methyl bromide (Sigma Chemical; $0.2 \mathrm{mg}$ in $0.3 \mathrm{ml}$ of $0.9 \%$ saline) and Chloropent (Fort Dodge Laboratories; $2.2 \mathrm{ml} / \mathrm{kg}$ ) intraperitoncally. When ancsthetized, the rat was placed in a stereotaxic instrument with the incisor bars in the same plane as the ear bars. The scalp was incised and retracted. Holes were drilled through the skull in the appropriate locations, and the lesion was made. The scalp sutured, and the rat received $0.1 \mathrm{ml}$, i.m., of Bicillin (Wyeth Laboratories) in both hind legs. The rat was removed from the stereotaxic instrument and placed on a paper towel in its home cage.

Lesions included the NBM and MSA. Ibotenic acid $(4.0 \mu \mathrm{g} / \mu \mathrm{l}$ in phosphate-buffered saline, $\mathrm{pH}$ 7.4) was microinjected at the following coordinates: MSA lesions $(n=10), 0.8 \mathrm{~mm}$ anterior to bregma, at the midline, and $5.8 \mathrm{~mm}$ ventral from the dura; NBM lesions $(n=10), 0.8$ $\mathrm{mm}$ posterior to bregma, $2.6 \mathrm{~mm}$ lateral to the central sinus, and 6.9 $\mathrm{mm}$ ventral from the dura. The infusion volume was $1.0 \mu \mathrm{l}$ for each injection site, and was delivered at a rate of approximately $0.1 \mu \mathrm{l} / \mathrm{min}$. The needle was left in place for approximatcly $5 \mathrm{~min}$ after the infusion.

FC lesions $(n=10)$ were made by aspiration of the cortex within the area circumscribed by the following coordinates: between 3 and $1.0 \mathrm{~mm}$ anterior to bregma, and between 1.0 and $4.0 \mathrm{~mm}$ lateral to the central sinus. The cingulate cortex was left intact, and an attempt was made to spare the underlying fiber tracts.

Control $(\mathrm{CON})$ rats $(n=10)$ received a variety of operations that did not damage the FC, NBM, or MSA. For some rats, the needle was lowered into the brain at coordinates appropriate to, but stopping just dorsal to the structure. For others, the skull was removed within the area described above for the FC lesions. Each rat's scalp was closed, and the animal was given Bicillin, as described above.

Postoperative PI retraining (sessions $31-38$ ). Rats began training again $7 \mathrm{~d}$ following surgery. The conditions of training were identical to those of preoperative sessions $1-30$.

Postoperative PI-GAP testing (sessions 39-66). The procedure was the same as the standard PI procedure, described above, with the following exceptions: During a random half of the peak trials, a single gap occurred in the signal. For these gap trials, the durations of the signal prior to the gap (S) and the durations of the gap $(G)$ were varied in a $2 \times 2$ factorial design within these sessions. The durations of $\mathbf{S}$ were 10 and $20 \mathrm{sec}$; the durations of $\mathrm{G}$ were 5 and $10 \mathrm{sec}$. The 4 different combinations of $S$ and $G$ occurred randomly, with equal probability. Data were collected from peak trials and gap trials.

Biochemical analysis. After testing, all rats were decapitated. The frontal neocortex (except for rats with FC lesions) and entire hippocampus were rapidly dissected on ice and stored at $-40^{\circ} \mathrm{C}$. The tissues were homogenized in $0.5 \%$ Triton X-100-10 mM EDTA, pH 7.4, and assayed for choline acctyltransferase (ChAT) activity (Fonnum, 1969). Protein 


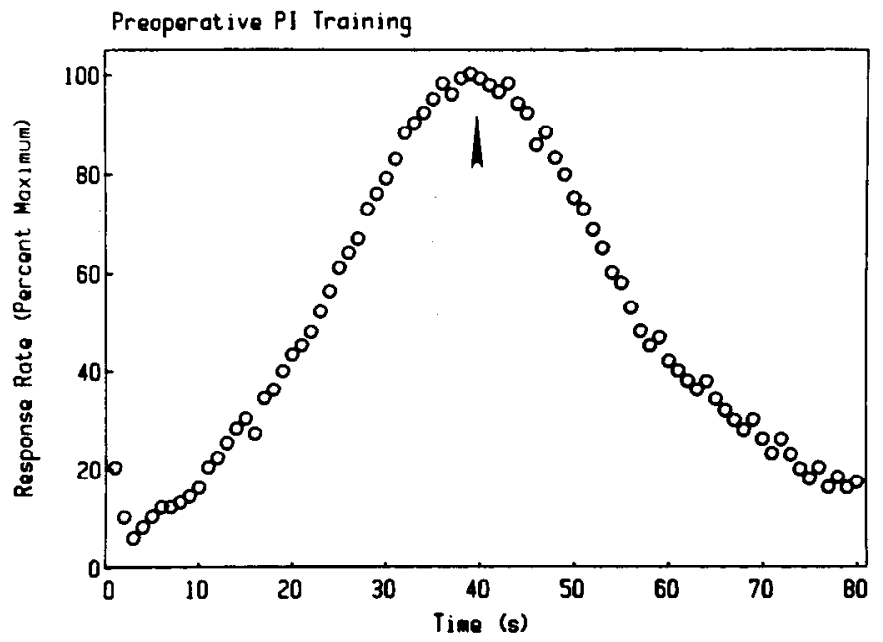

Figure 1. Preoperative behavior. Median response rate as a function of signal duration for all 40 rats during the last $7 \mathrm{~d}$ (sessions 24-30) of bascline peak-interval training. The arrow indicates the programmed time of reinforcement.

was measured according to Lowry et al. (1951). The frontal neocortical sample $\left(4 \mathrm{~mm}^{2}\right.$; approximately $\left.50-75 \mathrm{mg}\right)$ was taken from a region immediately lateral to the cingulate cortex in the motor cortex, areas 2,5 , and 10 .

Histology. After the removal of samples for biochemical analysis, the remaining brain tissue from each rat was fixed in a solution of $10 \%$ formalin and $30 \%$ sucrose, frozen, and sectioned coronally at $30 \mu \mathrm{m}$ with a frozen-stage microtome. Every fifth section throughout the lesion site was mounted on a glass slide and stained with cresyl violet. The size and location of the lesions were determined by microscopic examination for loss of magnocellular neurons and the presence of gliosis.

Data analysis. For each peak and gap trial, the number of responses in each $1 \mathrm{sec}$ interval during the $130 \mathrm{sec}$ signal was recorded for each rat. These functions were summed over trials for each session, and the mean response rate, as a function of time since signal onset, was calculated for each rat for each session. The mean response rate was then calculated for a specified number of sessions during each condition for each rat. The functions for individual rats were combined as follows: The response rate as a function of time since signal onset during the last 7 sessions was converted to the percentage of maximum response rates for each rat, and an average of these functions for the 10 rats was calculated. This measure equalizes the contribution of rats with different average response rates and has been used previously to describe timing functions (Meck and Church, 1984).

For the mean individual response rate functions, the peak time was estimated in the following manner: (1) The number of responses in the $6 \mathrm{sec}$ interval following each second was counted; (2) the interval with the maximum number of responses was determined; (3) the middle of that interval was recorded; and (4) if more than one interval satisfied the above criterion, the median value of these intervals was defined as the time of the peak rate on that trial. The peak rate was obtained by taking the median response rate during the selected $6 \mathrm{sec}$ interval(s).

All data were analyzed using Student's 2-tailed $t$ test and an analysis of variance (ANOVA) with post hoc Tukey contrasts.

\section{Results}

\section{Preoperative behavior}

PI baseline training (sessions 24-30). During PI baseline training, the peak time and peak rate measures were similar for all groups, $F(3,36)<1.0$, n.s. For all rats, the median and median average deviation for peak time was $40.5 \pm 0.5 \mathrm{sec}$, and the median peak rate was $59.7 \pm 5.6$ responses $/ \mathrm{min}$. The median response rate function for all 40 rats during the last $7 \mathrm{~d}$ of baseline training is shown in Figure 1. Numbers are medians \pm mcdian absolute deviation.
Postoperative PI Retraining

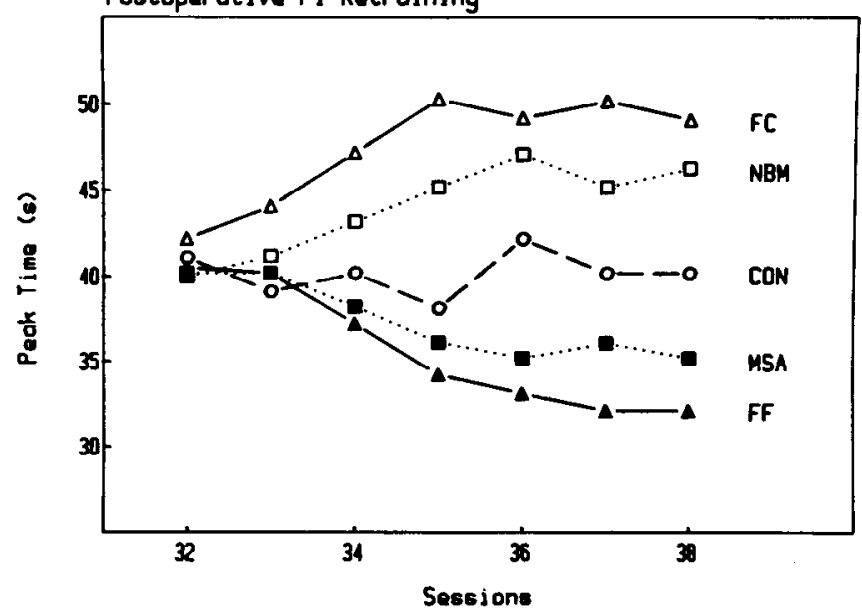

Figure 2. Postoperative behavior. Median peak times as a function of the first $7 \mathrm{~d}$ (sessions 32-38) of peak-interval retraining for rats with control operations $(C O N)$, lesions of the frontal cortex $(F C)$, of the nucleus basalis magnocellularis $(N B M)$, of the medial septal area $(M S A)$, and of the fimbria-fornix $(F F)$. The data for the FF group were adapted from Olton et al. (1987a).

\section{Postoperative behavior}

PI retraining (sessions 32-38). The daily median peak time response measure for the first $8 \mathrm{~d}$ of postoperative testing is presented for all groups in Figure 2. The behavioral data from session 31 were excluded from analysis because of some initial disruption following the recovery period. For comparison, data obtained in a prior experiment (Olton et al., 1987a) for rats with FF lesions have been rescaled and are presented in Figure 2.

ANOVAs showed a significant effect of training sessions for all groups except CON, $p<0.001$. During the last 3 sessions, the groups were ordered from the shortest to the longest peak time as follows: FF, MSA, CON, NBM, and FC. All adjacent paired comparisons were statistically different $(p s<0.05)$.

Peak time data from the last 3 sessions of postoperative retraining were analzyed for trial-to-trial regulation in peak time. The percentage of alternations or runs around the median peak time has been used as an index of the regulation of peak time (Meck, 1987). Typically, normal rats regulate their trial-to-trial peak times in a manner such that successive estimates of the time of reinforcement are not independent, but show a negativcly corrclatcd oscillation between a lower and upper limit that requires the use of working memory. In the present experiment, 3 groups had very similar regulation measures, all of which were significantly $(p<0.01)$ above the level of chance $(50 \%)$ : CON, $57.5 \pm 6 \%$; NBM, $58.7 \pm 6 \%$; and FC, $56.9 \pm$ $6 \%$. The remaining MSA group had a significantly $(p<0.001)$ lower regulation measure, $39.8 \pm 6 \%$, which was significantly $(p<0.01)$ below the level of chance.

Early PI-GAP testing, peak trials (sessions 39-45). The left column of Table 1 shows the median peak time for peak trials during the first $7 \mathrm{~d}$ of $40 \mathrm{sec}$ PI-GAP testing for all groups of rats. The median peak time of $\mathrm{CON}$ rats was not significantly different from the median preoperative peak time, $t(9)<1$. The median peak time of rats with FC and NBM lesions was significantly longer than the median preoperative peak time, $t(9)=$ $5.1, p<0.001$, and $t(9)=3.4, p<0.01$, respectively. In contrast, the median peak time of rats with FF and MSA lesions was 
Table 1. Peak time(s) in peak trials of the PI-GAP procedure early (sessions 39-45) and late (sessions 60-66) in testing

\begin{tabular}{llll} 
Group & Early & Late & Change \\
\hline CON & $49.5 \pm 5$ & $51.0 \pm 5$ & +1.5 \\
FC & $59.0 \pm 6$ & $58.5 \pm 7$ & -0.5 \\
NBM & $54.5 \pm 7$ & $51.5 \pm 5$ & -3.0 \\
MSA & $46.0 \pm 6$ & $49.5 \pm 6$ & +3.5 \\
FF & $41.0 \pm 5$ & $41.5 \pm 6$ & +0.5
\end{tabular}

Numbers are medians \pm median absolute deviation*. CON, control; $F C$, frontal cortex; NBM, nucleus basalis magnocellularis; MSA, medial septal area; FF, fimbriafornix. The data from rats with FF lesions are adapted from Olton et al. (1987a).

* Rounded to the nearest 0.5 .

significantly shorter than the median preoperative peak time, $t(9)=5.7, p<0.001$, and $t(9)=3.6, p<0.01$, respectively. There were no reliable differences in peak rate between groups.

Early PI-GAP testing, gap trials (sessions 39-45). During the first $7 \mathrm{~d}$ of 40 sec PI-GAP testing, the peak time for gap trials was compared to the peak time for peak trials. A summary of the median increase in peak time as a function of the duration of $\mathrm{S}$ (the signal prior to a gap) and of $\mathrm{G}$ (the gap) is presented in the top part of Table 2 (Early).

A 2-way ANOVA with repeated measures for factors $\mathrm{S}$ and $G$ showed that for CON, FC, and NBM, only the duration of $G$ had a significant effect on peak time, $F s(1,9)<3.36, p\rangle$ 0.10 and $F(1,9)>22.9, p<0.001$, respectively. For MSA rats, the duration of both $\mathrm{S}$ and $\mathrm{G}$ had a significant effect on peak time, $F(1,9)>2.29, p<0.001$.

A comparison of peak rate measures for peak trials and gap trials revealed no significant differences for any of the groups. The median peak rate was $63.4 \pm 7.5$ responses $/$ min on trials without gaps, and $60.1 \pm 5.6$ responses $/ \mathrm{min}$ on trials with gaps.

Late PI-GAP testing, peak trials (sessions 60-66). The right part of Table 1 (Late) shows the median peak time during the last $7 \mathrm{~d}$ of postoperative $40 \mathrm{sec}$ PI-GAP testing for the 4 groups of rats. The median peak time of rats with $\mathrm{CON}$ operations was not significantly different from the median preoperative peak time, $t(9)<1$. The median peak time of rats with FC lesions was significantly longer than the median preoperative pcak timc, $t(9)=4.8, p<0.001$. The median peak time of rats with NBM and MSA lesions was not significantly different from the median preoperative peak time, $t(9)=1.6, p>0.1$ and $t(9)=1.1, p>$ 0.2 , respectively. There were no reliable differences in peak rate between groups.

Late PI-GAP testing, gap trials (sessions 60-66). During the last $7 \mathrm{~d}$ of postoperative $40 \mathrm{sec}$ PI-GAP testing, the peak time for gap trials was compared to the peak time for peak trials without gaps. A summary of the increase in peak time as a function of the $S$ and $G$ intervals is presented in the bottom rows of Table 2 (Late).

A 2-way ANOVA with repeated measures for factors $S$ and G showed that for CON, FC, and NBM rats only the duration of $\mathrm{G}$ had a significant effect on peak time, $F s(1,9)<1.51, p\rangle$ 0.25 and $F_{S}(1,9)>22.9, p<0.001$, respectively. For MSA rats, the duration of both $\mathrm{S}$ and $\mathrm{G}$ had a significant effect on peak time, $F s(1,9)>22.9, p<0.001$.

A comparison of peak rate measures for peak trials and gap trials revealed no significant differences for any of the groups. The median peak rate was $64.6 \pm 6.7$ responses $/ \mathrm{min}$ for peak trials and $61.7 \pm 5.8$ responses $/$ min for gap trials.
Table 2. Increase in peak time(s) as a function of signal variables for GAP trials in the PI-GAP procedure

\begin{tabular}{|c|c|c|c|c|}
\hline \multirow[b]{3}{*}{ Group } & \multicolumn{4}{|c|}{ S: Duration of signal prior to gap } \\
\hline & \multicolumn{2}{|c|}{ G: Duration of gap } & \multicolumn{2}{|c|}{ G: Duration of gap } \\
\hline & $(5 \mathrm{sec})$ & $(10 \mathrm{sec})$ & $(5 \mathrm{sec})$ & $(10 \mathrm{sec})$ \\
\hline \multicolumn{5}{|c|}{ Early (sessions 39-45) } \\
\hline $\mathrm{CON}$ & $6.0 \pm 0.5$ & $10.5 \pm 0.5$ & $6.5 \pm 0.5$ & $10.5 \pm 0.5$ \\
\hline FC & $5.5 \pm 0.5$ & $11.5 \pm 1.0$ & $6.0 \pm 0.5$ & $11.0 \pm 0.5$ \\
\hline NBM & $5.5 \pm 0.5$ & $11.0 \pm 0.5$ & $6.0 \pm 0.5$ & $10.5 \pm 0.5$ \\
\hline MSA & $15.0 \pm 1.0$ & $20.5 \pm 1.0$ & $24.5 \pm 1.0$ & $28.5 \pm 2.5$ \\
\hline \multicolumn{5}{|c|}{ Late (sessions 60-66) } \\
\hline $\mathrm{CON}$ & $6.0 \pm 0.5$ & $11.0 \pm 0.5$ & $5.0 \pm 0.5$ & $11.5 \pm 0.5$ \\
\hline FC & $5.5 \pm 0.5$ & $10.5 \pm 0.5$ & $5.5 \pm 0.5$ & $11.5 \pm 0.5$ \\
\hline NBM & $5.5 \pm 0.5$ & $10.5 \pm 0.5$ & $6.5 \pm 0.5$ & $10.5 \pm 0.5$ \\
\hline MSA & $14.5 \pm 1.0$ & $21.5 \pm 1.0$ & $26.0 \pm 1.0$ & $30.5 \pm 2.5$ \\
\hline
\end{tabular}

Numbers are medians \pm median absolute deviation, rounded to the nearest 0.5 . CON, control; FC, frontal cortex; NBM, nucleus basalis magnocellularis; MSA, medial septal area.

Biochemistry. The levels of ChAT activity in the rats with ibotenic acid (IBO) lesions were compared to the values in CON rats to determine the percentage decrease in activity caused by the lesions (see Table 3 ). MSA lesions significantly decreased the levels of ChAT activity in the hippocampus $(p<0.01)$, but not in the frontal cortex. By contrast, NBM lesions significantly decreased the levels of ChAT activity in the frontal cortex $(p<$ 0.01 ), but not in the hippocampus.

Histology. Lesions of the MSA destroyed most of the cells of the medial septum and the dorsal part of the vertical limb of the diagonal band. Lesions of the NBM were centered in the substantia innominata and extended $2.0 \mathrm{~mm}$ caudally from the anterior commissure. Elsewhere, we have documented in detail the extent of cell loss in the basal forebrain, the morphology of the lesions, and the changes in biochemistry that occur in the projection areas (Wenk and Olton, 1984; Wenk et al., 1984, 1987a; Hepler et al., 1985a, b). Consequently, that information is not repeated here. FC aspirations removed the frontal cortex, sparing the cingulate cortex and the underlying fiber tracts of the corpus callosum. A typical FC lesion is shown in Figure 3.

\section{Discussion}

The differential effects of lesions placed in the NBM and the FC, on the one hand, and in the MSA and the FF, on the other, show that both of these neural systems are involved in temporal memory, but in complementary ways. The dissociations between these systems are seen in 3 measures. First, in peak trials, the NBM and FC lesions produced an increase (a rightward shift) in the peak time, while the MSA and FF lesions produced

Table 3. ChAT levels in frontal cortex and hippocampus

\begin{tabular}{lll} 
& \multicolumn{2}{l}{ ChAT $(\mathrm{nmol} / \mathrm{mg}$ of protein $/ \mathrm{hr})$} \\
\cline { 2 - 3 } Group & Frontal cortex & Hippocampus \\
\hline CON & $23.4 \pm 2.1$ & $32.1 \pm 3.5$ \\
NBM & $14.2 \pm 1.4$ & $27.2 \pm 2.0$ \\
MSA & $19.3 \pm 2.1$ & $24.2 \pm 2.6$
\end{tabular}

Numbers are medians \pm median absolute deviation. CON, control; NBM, nucleus basalis magnocellularis; MSA, medial septal area. 


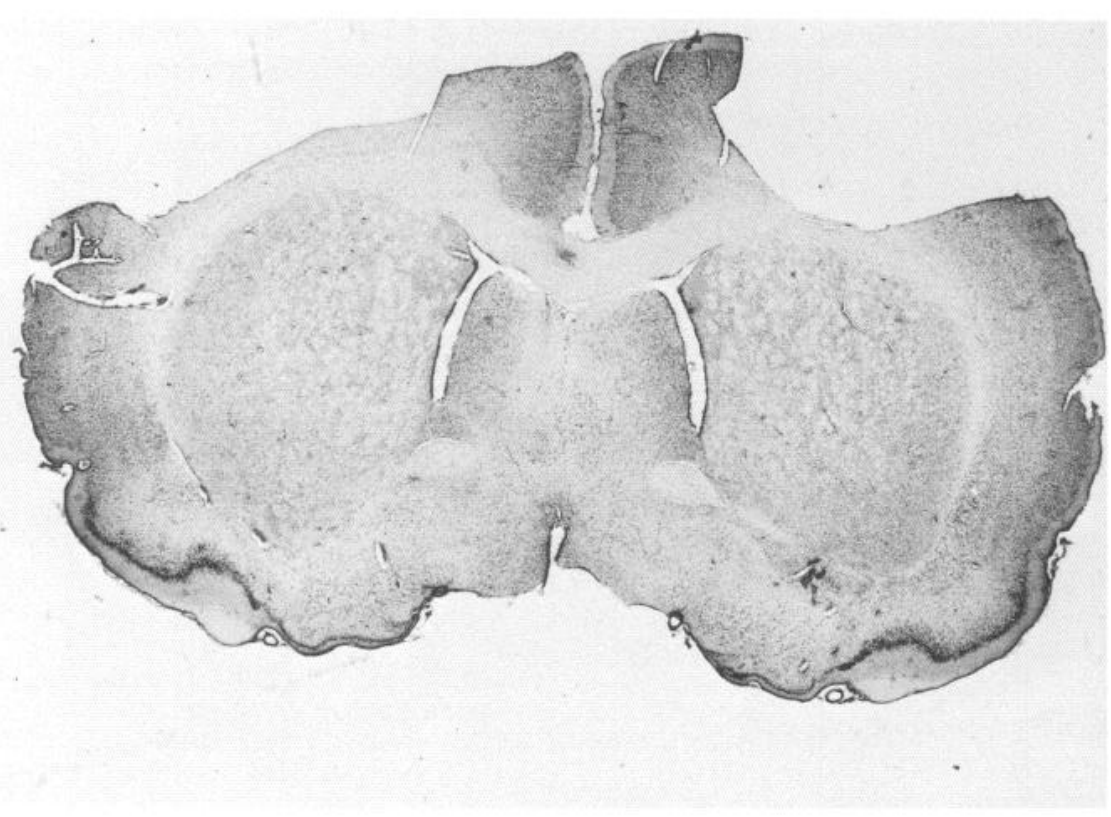

Figure 3. Histological result for a representative rat with a large frontal cortex lesion. The brain was stained with cresyl violet stain for cell bodies and luxol fast blue for myelinated fiber tracts. a decrease (a leftward shift) in the peak time. Second, the MSA and FF lesions produced a massive reduction in the regulation of the temporal criterion used on successive peak trials, indicating a failure in the ability to regulate, while the lesions in the NBM and FC produced no change. Third, in the gap trials, the MSA and FF lesions produced resetting, evidence of a failure of working memory, while the lesions in the NBM and FC produced no change. Each of these points will be discussed below.

The leftward shift in the peak time following MSA lesions was in the same direction as that produced by FF lesions (Meck et al., 1984, 1986b; Olton et al., 1987a, b). These data demonstrate that destruction of the projections from the MSA to the hippocampus is able to modify the memory of the time of reinforcement in reference memory. This modification suggests that damage to these fibers may have been responsible for the leftward shift seen in rats with FF lesions. Because a proportional leftward shift in peak times implies an increase in activity of those mechanisms directly involved in the storage of information in reference memory, damage to the MSA must produce a concomitant increase in activity in other, interrelated brain regions that are responsible for coding the remembered time of reinforcement (Meck and Church, 1987a).

The lesions in the NBM and FC produced a rightward shift of the peak time. These lesions affected the remembered time of reinforcement, but in a direction opposite to that from lesions in the MSA and FF (Meck et al., 1984, 1986b; Olton et al., $1987 \mathrm{a}, \mathrm{b})$. The rightward shift in peak time is consistent with a decrease in activity of those mechanisms responsible for the storage of temporal information in reference memory. Because lesions in the NBM and FC decreased the speed of memory storage, there must be a direct relation between neural activity in the frontal system and the content of temporal memory. Evidence of cholinergic activity in the frontal cortex, as measured by sodium-dependent high-affinity choline uptake indicates a similar relation between this brain region and the content of temporal reference memory (Meck et al., 1986a).

Rats with MSA lesions, but not with NBM or FC lesions, showed significantly fewer runs in their successive peak time measures than did CON rats. The number of crossings of the median peak time for successive peak time measures (runs) is an indicator of whether the sampling of the temporal criterion used on each trial is controlled in some way by behavior on previous trials or is random. CON, NBM, and FC rats showed control of the temporal criterion that they used on each trial by alternating this value between an upper and lower limit. This regulation of the value of the temporal criterion used on successive trials produced more than the expected number of runs for successive peak trial observations. This effect was not due to the random insertion of food trials, since an analysis of the peak time on peak trials immediately following a food trial showed no reliable effect of reinforcement on the location of the peak. In contrast, rats with MSA lesions showed no evidence of regulating the temporal criteria they used on successive trials. The sequence of temporal criteria used by rats with MSA lesions was not completely random, however. There were systematic drifts in successive peak time values that led to the observation of fewer than the expected number of runs for successive peak trials. This effect was not due to the random insertion of food trials because an analysis of the peak time on peak trials immediately following a food trial showed no reliable effect of reinforcement on the temporal location of the peak. Rats with FF and MSA lesions were apparently unable to regulate successive peak time values because of an enduring failure to remember the temporal criterion used on the previous trial, a value that should be stored in working memory.

The dissociation between the effects of NBM and MSA lesions on working and reference memory is underscored further by the observation that, following MSA lesions, the reference memory dysfunctions (peak time in peak trials) recovered, while the working memory dysfunctions (peak time in gap trials) and regulation of peak time values on a trial-to-trial basis did not.

The leftward and rightward shifts in peak time produced by FF lesions (Meck et al., 1984, 1986b; Olton et al., 1987a, b) and FC lesions, respectively, were apparently permanent and showed no signs of recovery during the experiment. Consequently, these manipulations caused a long-term effect in the memory storage mechanisms used to process temporal infor- 
mation. Although the leftward and rightward shifts produced by lesions of the MSA and NBM developed during testing in a manner similar to the shifts produced by $F F$ and FC lesions, they were transitory in nature and showed nearly complete recovery with extended training. This normalization of behavior in MSA and NBM rats may reflect relearning, using the undamaged portions of the system in which the lesion was made or compensatory processes produced by a variety of mechanisms involving the damaged system and others. Studies designed specifically to examine the mechanisms of recovery of function will be necessary to distinguish among these various alternatives.

The pattern of changes in timing following all of these lesions indicates that the changes in peak time are a result of an alteration of memory storage, rather than of the pacemaker mechanism of the internal clock. As detailed elsewhere (Meck, 1983, 1986; Church, 1984; Church and Meck, 1987; Meck and Church, 1987a, b), manipulations that affect the internal clock result in an immediate shift of peak time that is gradually corrected and returned to normal with experience. In contrast, alterations of memory storage produce the pattern observed here: little or no immediate effect after treatment, and a gradual development of a relatively permanent shift in peak time. Consequently, the effects of the lesions are interpreted as producing alterations in temporal reference memory, rather than in the clock mechanism itself.

The FF lesions (Olton et al., 1987a) and the MSA lesions produced a marked change in behavior during the gap trials. $\mathrm{CON}$ rats followed a stop rule during the gap. These rats stopped timing the signal during the gap, remembered the duration of the signal prior to the gap, and added the duration of the signal prior to the gap to the duration of the signal following the gap. Consequently, their peak times were shifted to the right by an interval equal to the gap, as compared to peak trials without a gap. For example, if there was a $10 \mathrm{sec}$ signal before the gap and a $5 \mathrm{sec}$ gap, the time of the peak was increased by about 5 sec (see Table 2). In contrast, rats with FF (Olton et al., 1987a) and MSA lesions followed a reset rule. When a gap occurred in the signal, these rats forgot the duration of the signal prior to the gap and began timing after the gap as if the preceding signal had never occurred. Consequently, their peak times were shifted to the right by an interval equal to the duration of the gap plus the signal prior to the gap. For example, if there was $10 \mathrm{sec}$ of signal before the gap and a $5 \mathrm{sec}$ gap, the time of the peak was increased by about $15 \mathrm{sec}$ (see Table 2). The gap procedure did not disrupt timing in general, which would have been shown by irregularities in the peak time function or an increase in its variability. The form and spread of the peak time functions relative to the mean (see Gibbon et al., 1984) following gaps were indistinguishable from those preceding the gap; only the position of the peak time was altered by the lesions. Thus, these lesions affected a specific memory system, rather than disrupting behavior in general. For rats with MSA lesions, resetting of the internal clock as a result of a gap in the signal continued throughout postoperative testing, even after the leftward shift in peak time had disappeared. Consequently, the resetting in gap trials was dissociable from the leftward shift in peak trials, suggesting that the MSA may have a more fundamental role in the working memory mechanism required by gap trials than in the reference memory mechanism used in peak trials.

In summary, the frontal system plays a major role in reference memory, as indicated by the location of the peak time for peak trials. In contrast, the hippocampal system plays a major role in working memory, as indicated by the resetting of the internal clock on pcak trials with gaps and by the failure to regulate trialto-trial peak times. These selective effects of lesions in different brain systems demonstrate the value of combining neural analysis with a formal psychological analysis of timing behavior. With the application of a timing model developed to describe normal timing behavior, information has been obtained about the neural mechanisms involved in the pacemaker, the reference memory for the remembered times of reinforcement, and the working memory for the duration of a currently timed event. Further analysis of normal animals and subjects with timing dysfunctions should be able to provide the specific details of how the neural systems code the time dimension.

\section{References}

Church, R. M. (1984) Properties of the internal clock. Ann. NY Acad. Sci. 423: 566-582

Church, R. M., and W. H. Meck (1987) Biological basis of the remembered time of reinforcement. In Quantitative Analysis of Behavior: Biological Determinants of Reinforcement, vol. 7, M. L. Commons, R. M. Church, J. R. Stellar, and A. R. Wagner, eds., Erlbaum, Hillsdale, NJ (in press).

Fuster, J. M. (1980) The Prefrontal Cortex, Raven, New York.

Gibbon, J., R. M. Church, and W. H. Meck (1984) Scalar timing in memory. Ann. NY Acad. Sci. 423: 52-77.

Hepler, D. J., B. L. Cribbs, D. S. Olton, and J. T. Coyle (1985a) Memory impairments following basal forebrain lesions. Brain Res. 346: 9-14.

Hepler, D. J., D. S. Olton, G. L. Wenk, and J. T. Coyle (1985h) I esions in nucleus basalis magnocellularis and medial septal area of rats produce qualitatively similar memory impairments. J. Neurosci. 5: 866873 .

Kolb, B. (1984) Functions of the frontal cortex of the rat: A comparative review. Brain Res. Rev. 8: 65-98.

Lowry, O. H., N. Rosebrough, A. Farr, and R. Randall (1951) Protein measurement with the Folin phenol reagent. J. Biol. Chem. 193: 265276.

Maricq, A. V. (1978) Some effects of lesions of the prefrontal cortex on timing behavior in the rat. Unpublished honors thesis, Brown University.

Meck, W. H. (1983) Selective adjustment of the speed of internal clock and memory storage processes. J. Exp. Psychol. [Anim. Behav.] 9: $171-201$.

Meck, W. H. (1986) Affinity for the dopamine $D_{2}$ receptor predicts neuroleptic potency in decreasing the speed of an internal clock. Pharmacol. Biochem. Behav. 25: 1185-1189.

Meck, W. H. (1987) Hippocampal function is required for feedback control of an internal clock's criterion. Behav. Neurosci. (in press).

Meck, W. H., and R. M. Church (1984) Simultaneous temporal processing. J. Exp. Psychol. [Anim. Behav.] 10:1-29.

Meck, W. H., and R. M. Church (1987a) Cholinergic modulation of the content of temporal memory. Behav. Neurosci. 101: 457-464.

Meck, W. H., and R. M. Church (1987b) Nutrients that modify the speed of internal clock and memory storage processes. Behav. Neurosci. 101: 465-475.

Meck, W. H., R. M. Church, and D. S. Olton (1984) Hippocampus, time, and memory. Behav. Neurosci. 98: 3-22.

Meck, W. H., R. M. Church, G. L. Wenk, and D. S. Olton (1985) Ibotenic acid lesions of the medial septal area and nucleus basalis magnocellularis cause differential impairments in temporal memory. Soc. Neurosci. Abstr. 11: 332.

Meck, W. H., R. M. Church, and G. L. Wenk (1986a) Arginine vasopressin inoculates against age-related increases in sodium-dependent high affinity choline uptake and discrepancies in the content of temporal memory. Eur. J. Pharmacol. 130: 327-331.

Meck, W. H., R. M. Church, G. L. Wenk, and D. S. Olton (1986b) Simultaneous temporal processing and the basal forebrain cholinergic system. Soc. Neurosci. Abstr. 12: 750 .

Milner, B. (1982) Some cognitive effects of frontal-lobe lesions in man. Phil. Trans. R. Soc. Lond. [Biol.] 298: 211-226.

Mitchell, S. J., J. N. P. Rawlins, O. Steward, and D. S. Olton (1982) Medial septal lesions disrupt theta rhythm and cholinergic staining 
in medial entorhinal cortex and produce impaired radial arm maze behavior in rats. J. Neurosci. 2: 292-302.

O'Keefe, J., and L. Nadel (1978) The Hippocampus as a Cognitive Map, Clarendon, Oxford, UK.

Olton, D. S., J. T. Becker, and G. E. Handelmann (1979) Hippocampus, space, and memory. Behav. Brain Sci. 2: 313-322.

Olton, D. S., W. H. Meck, and R. M. Church (1987a) Separation of hippocampal and amygdaloid involvement in temporal memory dysfunctions. Brain Res. 404: 180-188.

Olton, D. S., G. L. Wenk, R. M. Church, and W. H. Meck (1987b) Attention and the frontal cortex as examined by simultaneous temporal processing. Neuropsychologia (in press).

Olton, D. S. G. L. Wenk, and D. J. Hughey (1987c) Dementia: Animal models of the cognitive impairments produced by degeneration of the basal forebrain cholinergic system. In Psychopharmacology: The Third Generation of Progress, R. Mohs, ed., Raven, New York (in press).

Rawlins, J. N. P. (1985) Associations across time: The hippocampus as a temporary memory store. Behav. Brain Sci. 8: 479-497.

Roberts, S. (1981) Isolation of an internal clock. J. Exp. Psychol. [Anim. Behav.] 7: 242-268.

Saper, C. B. (1984) Organization of cerebral cortical afferent systems in the rat. I. Magnocellular basal nucleus. J. Comp. Neurol. 222:313342.
Schwarcz, R., T. Hökfelt, K. Fuxe, G. Jonsson, M. Goldstein, and L. Terenius (1979) Ibotenic acid-induced neuronal degeneration: A morphological and neurochemical study. Exp. Brain Res. 37: 199216.

Sofroniew, M. V., F. Eckenstein, H. Thoenen, and A. C. Cuello (1982) Topography of choline acetyltransferase-containing neurons in the forebrain of the rat. Neurosci. Lett. 33: 7-12.

Wenk, G. L., and D. S. Olton (1984) Recovery of neocortical choline acetyltransferase activity following ibotenic acid injection into the nucleus basalis of Meynert in rats. Brain Res. 293: 184-186.

Wenk, G. L., B. Cribbs, and L. McCall (1984) Nucleus basalis magnocellularis: Optimal coordinates for selective reduction of choline acetyltransferase. Exp. Brain Res. 56: 335-340.

Wenk, G. L., D. J. Hughey, V. Boundy, A. Kim, L. Walker, and D. S. Olton (1987a) Neurotransmitters and memory: The role of cholinergic, serotonergic, and noradrenergic systems. Behav. Neurosci. 101: 325-332.

Wenk, G. L., D. J. Hughey, and D. S. Olton (1987b) Basal forebrain cholinergic neurons and Alzheimer's disease. In Experimental Models of Dementing Disorders: A Synaptic Neurochemical Perspective, J. Coyle, ed., Liss, New York (in press). 